\title{
Development of an effective way to increase the biological activity of nicotinamide - a new strategy to protect against photoageing and skin neoplasia
}

\author{
Elena Uspenskaya ${ }^{1 *}$, Tatyana Pleteneva ${ }^{1}$, Anton Syroeshkin ${ }^{1}$, Ilaha Kasymova ${ }^{1}$, and \\ Nataliya Zakharova ${ }^{2}$ \\ ${ }^{1}$ Peoples Friendship University of Russia (RUDN University), Department of Pharmaceutical and \\ Toxicological Chemistry, 117198, Miklukho-Maklaya str., 6, Moscow, Russian Federation \\ ${ }^{2}$ I.M. Sechenov First Moscow State Medical University (Sechenov University), Department of \\ Chemistry of the Institute of Pharmacy, 119991, Trubetskaya str., 8-2, Moscow, Russian Federation
}

\begin{abstract}
The objective of this work is to demonstrate changes in properties and increase of biological activity of nicotinamide (NAM) substance for skin protectants activated by mechanical activation (MA). To assess the physical, chemical and biological properties of NAM were used biotesting by the Spirotox-method, direct and indirect optical methods (microscopy, laser diffraction), infrared spectroscopy, pH-metry. The results of the study showed an increase in biological activity, expressed in changes in the energy of activation of cell transition to the "dead cell" state on the example of biosensor Spirostomum ambiguum. Also we indicated an increase in the rate of the chemical process of dissolution of mechanoactivated molecular crystals of nicotinamide (NAM) expressed in the values of the first order rate constant. Mechanical activation at a high rate of NAM substance powder deformation has led to changes in physical, chemical and biological properties of the drug, which can be used in medicine to increase efficiency and reduce doses of pharmacotherapy. Keywords: nicotinamide (NAM), basal cell carcinoma (BCC), mechanical activation (MA), microscopy, laser diffraction, Spirotox method.
\end{abstract}

\section{Introduction}

One of the ways to activate the biological and physical-chemical properties of powders of active pharmaceutical substances is fine grinding by mechanical action [1,2]. In a highly dispersed state, compounds become more chemically active, as fine grinding is accompanied by an increase in the free energy reserve and formation of centers with high activity on newly formed surfaces. The result of these processes is the accumulation of point defects, amorphous regions, dislocations, and disclinations in the structure of molecular crystals. Unbalanced states with highly stored deformation energy have a

\footnotetext{
* Corresponding author: uspenskaya75@mail.ru
} 
significant impact on physical and mechanical properties of materials [3]. The material can be crushed in various ways: crushing, breaking, striking, rubbing, cutting or a combination of these methods. Results on static and dynamic loading of powdery materials showed that of all the ways of destruction the most effective is "free and constrained impact" (fig. 1).

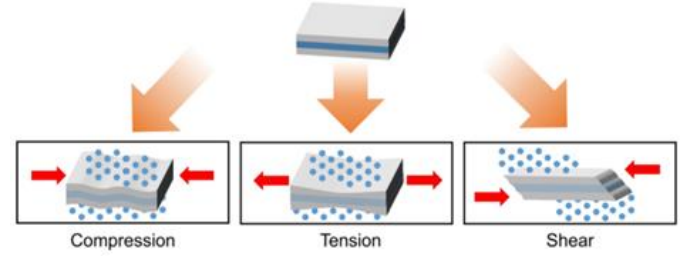

Fig. 1. Schematic representation of compressive, tensile, and shear forces [4].

Changing the structure of molecular crystals without changing their composition becomes particularly important for pharmacy, as it makes it possible to improve the pharmacological properties of API, for example, to significantly increase the rate of dissolution and solubility, and, consequently, the bioavailability of the drug [5, 6].

Due to the steady increase in the incidence of skin cancer, new methods of its treatment are relevant, associated with relatively satisfactory results of the arsenal of existing methods. In the world by 2025 , the incidence of skin cancer will increase by $25 \%$ [7]. Basal cell carcinoma (BCC) is a malignant neoplasm, which constitutes up to $75 \%$ of all epithelial malignant neoplasms of the skin and is characterized by selective localization on the skin of the head and neck [8]. Among the gentlest methods of BCC treatment specialists refer immunotherapy, photodynamic therapy, local chemotherapy, laser therapy, etc., capable of suppressing the main pathogenic pathways of tumor development. Prevention of malignant skin epithelial tumors (SET) consists in the protection of the skin from adverse radiation and ultraviolet effects, as well as chemoprophylaxis of SET. Research conducted by the authors [9] has shown a significant role in the chemoprophylaxis of skin cancer niacin/nicotinamide (NAM) are the forms of the B3 vitamin - coenzymes nicotinamide adenine dinucleotide (NAD) and nicotinamide adenine dinucleotide phosphate (NADP) in vivo (metabolic processes) [10]. In medicine, high-dose pharmacotherapy with nicotinamide is known and widely used. The therapeutic index of NAM is wide, but at very high doses in animals and humans, reversible hepatotoxicity was noted [11]. The $\mathrm{LD}_{50}$ of $\mathrm{NAM}$ in rats is $1,68 \mathrm{~g} / \mathrm{kg}$; the $\mathrm{LD}_{50}$ in mice is estimated as $4,5 \mathrm{~g} / \mathrm{kg}$ when given orally and $2,5 \mathrm{~g} / \mathrm{kg}$ when given intravenously. Other toxic effects associated with the use of high doses of NAM are bullous lesions of the skin, toxic amblyopia and hypotensive reactions [12]. Consequently, high doses of nicotine $(\leq 3 \mathrm{~g})$ should be considered as a therapy with toxic potential.

In this regard, a relevant issue is the study of factors contributing to the increase in bioavailability of a medicinal substance. To obtain drugs with high biological and pharmaceutical availability, in addition to other generally accepted methods [13], mechanical activation at high rate of deformation of API crystal structure is used [14].

\section{Materials and methods}

Active pharmaceutical ingredients Nicotinamide (NAM) produced by «G. AmphrayLab.», India.

The study of individual biological activity was carried out using the Spirostomum ambiguum test culture by Spirotox method, based on the establishment of Arenaius kinetics of dependence of reaction rates (time of cell biosensor death) on temperature.

The fine grinding of NAM was carried out by grinding in a mechanical cutting knife mill on the type of "free, direct impact" (by the Rumpf method) [15]. Mechanical 
processing of NAM powder was carried out within 20 minutes in impulse mode, i.e. with alternating processes of stress field generation and its relaxation during several days [16].

Particle size analysis of NAM powders before and after fine grinding was performed by direct optical microscopy using Altami BIO 2 microscope with 10x lens magnification.

The identification of the native and mechanically activated NAM powder was established by the method of IR-spectroscopy using Cary 630 IR-Fourier spectrometer (Agilent Technologies, USA).

The determination of the dissolution rates in water of the NAM substance powders was carried out with the use of the Malvern 3600 EC dispersion meter by means of a low-angle laser light scattering method [17].

$\mathrm{pH} 5 \%$ of the aqueous solutions of the NAM substance powder were measured according to the EU pharmacopoeia 8.0 [18], using a Sartorius $\mathrm{pH}$ meter.

\section{Results and discussion}

\subsection{Fine grinding and optical microscopy}

Dry grinding was carried out under the influence of shear and shearing forces (see fig. 1) in a batch knife mill. Figure 2 shows the appearance and particle size analysis of a NAM substance crystal before (a) and after (b) the fine grinding in a mechanical cutting knife.

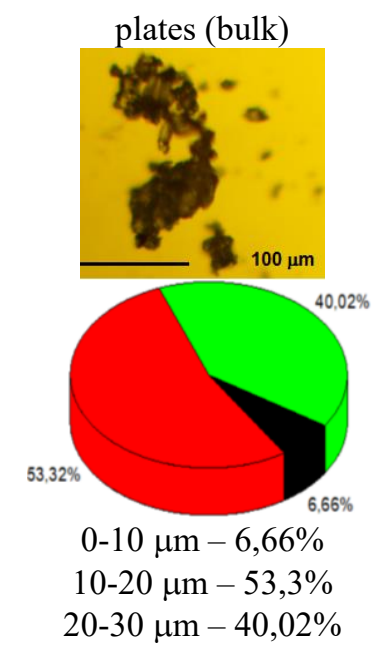

a

\section{Particle shape}

particles with chipped edges, uneven surface
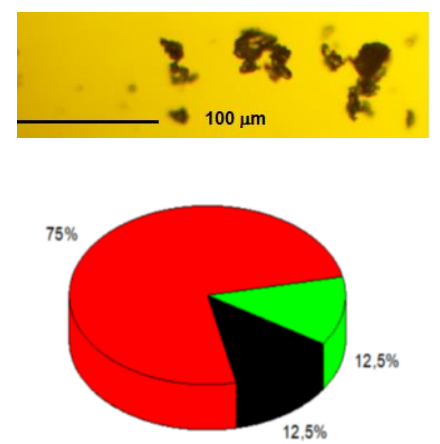

$$
\begin{array}{r}
0-10 \mu \mathrm{m}-12,5 \% \\
10-20 \mu \mathrm{m}-75,0 \% \\
20-30 \mu \mathrm{m}-12,5 \% \\
\mathrm{~b}
\end{array}
$$

Fig. 2. Appearance and size of NAM crystals from optical microscopy dates.

According to [19], part of the mechanical energy supplied to the solid at the time of activation is absorbed by it in the form of new surface, linear and point defects As can be seen (Fig.2), after fine crushing of the particles, chips, rough and uneven surfaces are formed. The number of particles up to $10 \mu \mathrm{m}$ in diameter doubled and the number of particles from 20 to $30 \mu \mathrm{m}$ tripled. With the help of mechanical activation as a result of fine grinding it is possible to use in chemistry the physical phenomena occurring in solids at high deformation rates: changes in the structure of solids; acceleration of dissolution and 
diffusion processes; formation of active centers on the freshly formed surface and related changes in biological activity of substances.

\subsection{IR-spectroscopy}

IR spectra before and after fine grinding were obtained and analyzed to confirm the identity of the experimental substance (Fig. 3).

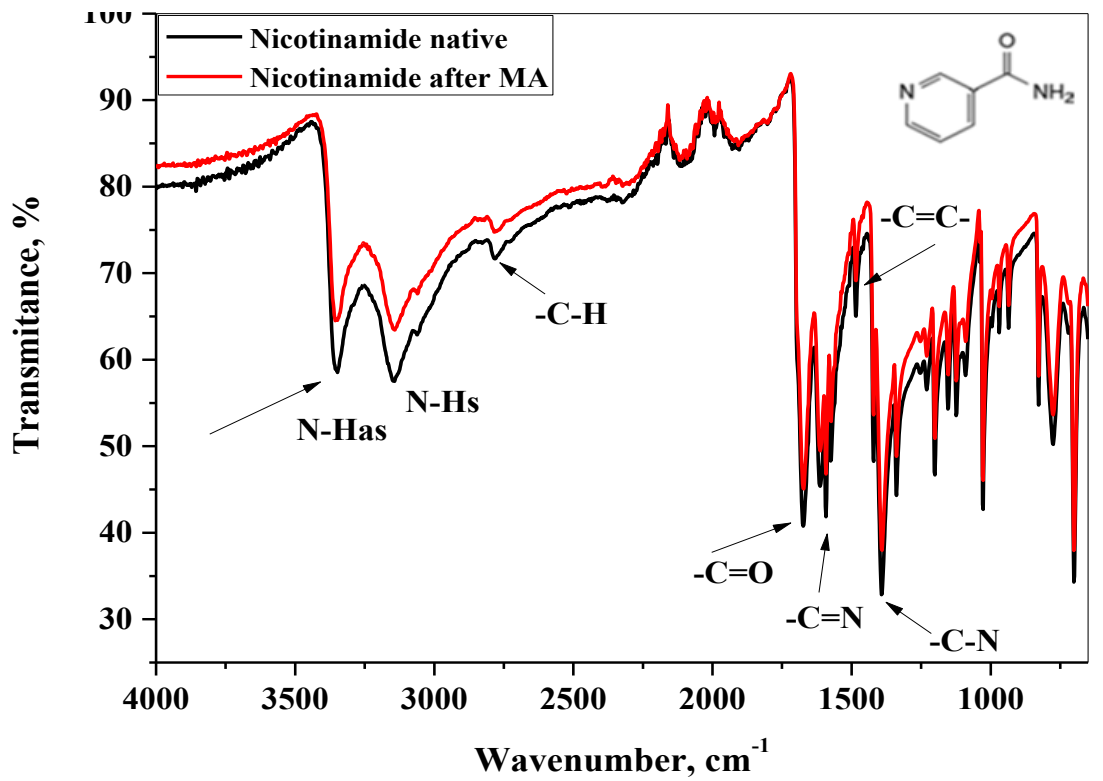

Fig. 3. The Fourier Transform Infrared spectroscopy (FTIR) spectra of the NAM substances before and after fine grinding process (mechanical activation, MA).

The presented infrared spectra are characterized by typical vibrational frequencies of bonds in nuclear groups: $v_{\text {as }} \mathrm{N}-\mathrm{H}$ at $3378 \mathrm{~cm}^{-1} ; v_{\mathrm{s}} \mathrm{N}-\mathrm{H}$ at $3109 \mathrm{~cm}^{-1} ; v \mathrm{C}-\mathrm{H}$ at $2750 \mathrm{~cm}^{-1}$; $v_{\text {as }}$ $\mathrm{C}=\mathrm{O}$ at $1700 \mathrm{~cm}^{-1}$; as well as out-of-plane $\left(\delta \mathrm{N}-\mathrm{H}\right.$ at $\left.860 \mathrm{~cm}^{-1}\right)$ vibrations in low frequency range. The found characteristic light transmission frequencies make it possible to identify nicotinamide. The coincidence of the FTIR curves in the position of the characteristic frequencies before and after the fine crushing of NAM indicates that the nature of the crystals is kept. Slight changes in the intensity of spectral maxima are observed, which may indicate a change in the quantitative content of the respective groups in a given substance. Consequently, all changes in the properties of API powder presented in this paper are caused by changes in the diameter of the powder particles (see Fig. 2) and the formation of centers with increased activity on newly formed surfaces, rather than by changes in the nature of the substance.

\subsection{Spirotox method}

The biological activity studies were carried out using the Spirostomum ambiguum test culture. The kinetic scheme of ligand-induced death of $S$. ambiguum includes the intermediate state $\left(\mathrm{C}-\mathrm{L}_{\mathrm{n}}\right)$, the stage of transition to the «dead cell» state (DC), which determines the rate of ligand-receptor interaction [20]. The existence of an intermediate 
state in the process of ligand-induced cell death of the test object means that the process of cell death should take place at the stage activation with energy consumption:

$$
k=A e^{-E a / T}
$$

From this point of view, it can be assumed that parameter $E_{a}$ is a quantitative criterion of the toxicity of aqueous solutions [5]. The study of temperature dependence of ligandinduced death of test objects in 5\% aqueous solutions of NAM before and after MA was carried out in the temperature range of $24 \div 32{ }^{\circ} \mathrm{C}$ with a step of $2{ }^{\circ} \mathrm{C}$. The exponential dependence of infusion death rate is typical for solutions of all investigated compounds, regardless of the produced mechanical action (fig. 4).
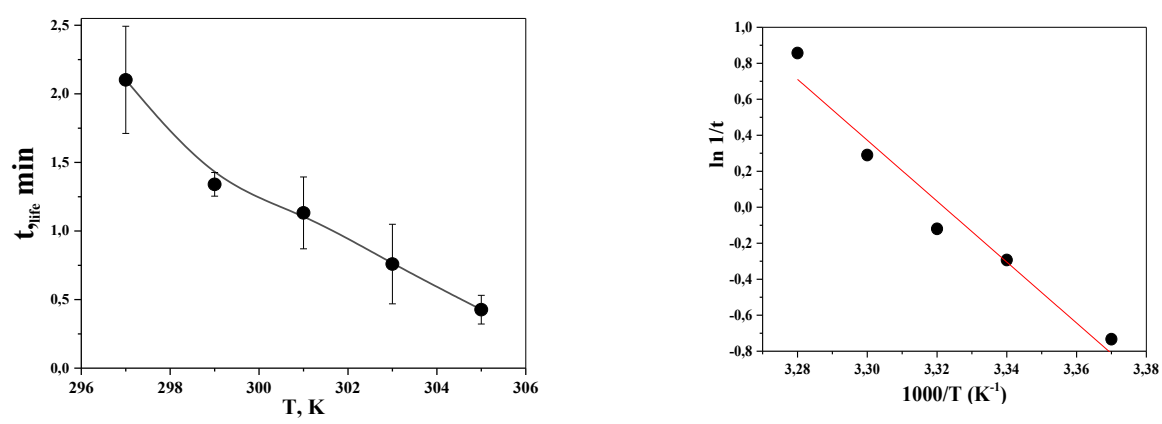

A
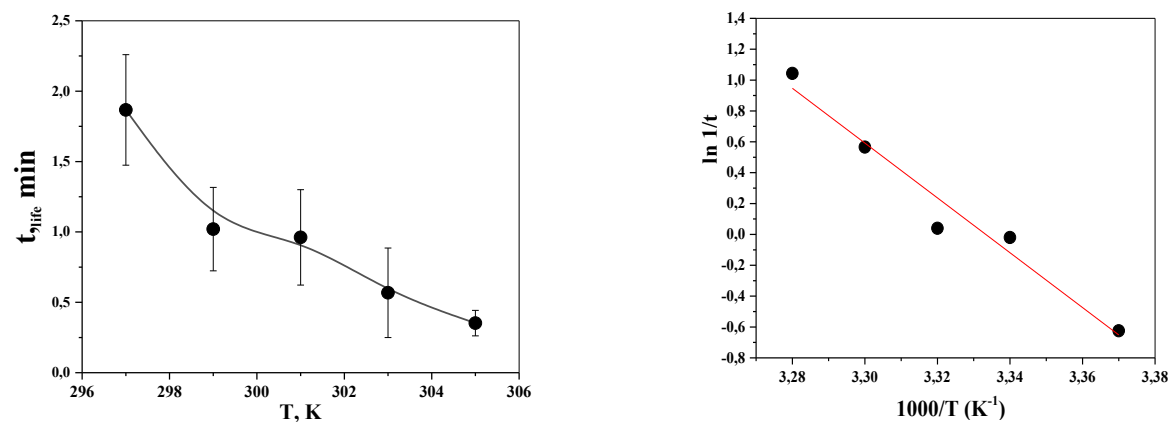

B

Fig. 4: Temperature dependency of cell biosensor life time process in 5\% aqueous solutions of NAM before (A) and after fine grinding process (B) in both direct and semi-log plots $(\mathrm{n}=5, \mathrm{P}=0,90)$.

To exclude the influence of $\mathrm{pH}$ of the aqueous solution on the lifetime of the cell biosensor, the control of the reaction of the medium was carried out using the EU pharmacopoeia method: $5 \%$ aqueous solutions of NAM have $\mathrm{pH}=6,5(\mathrm{n}=3)$.

The life time dependence on temperature of ligand-induced cellular transitions of $S$. ambiguum was found to be linearized in Arenius coordinates: $\lg (1 / \mathrm{t})-1000 / \mathrm{T}$. By the tangent to the angle of inclination of the lines in semi-logarithmic coordinates were found values of activation energy ( $\left.{ }^{\circ b s} \mathrm{E}_{\mathrm{a}}\right)$ of cell death in the solution of NAM before $(141 \mathrm{~kJ} / \mathrm{mol})$ and after $(120 \mathrm{~kJ} / \mathrm{mol}) \mathrm{MA}$. It is shown the influence of mechanochemical activation on the increase of biological activity of NAM to reduce the value of activation energy of cell death by $20 \mathrm{~kJ} / \mathrm{mol}$. After fine grinding the free surface is not equilibrium and statically stable in the surface layer the processes of rearrangement towards the equilibrium state begin. 
Arrangement of the structure (transition "order-before-order-new order") significantly depends on the temperature of the system as a whole.

\subsection{Rate of dissolution}

Bioavailability of oral drugs depends on their ability to absorb and the degree of its penetration through the wall of the gastrointestinal tract. Increasing the rate of dissolution of drugs will increase their bioavailability. We investigated the process of NAM dissolution water before and after fine grinding by laser diffraction method based on the dependence of light scattering on the concentration of disperse phase particles in the water suspension (fig. 5).

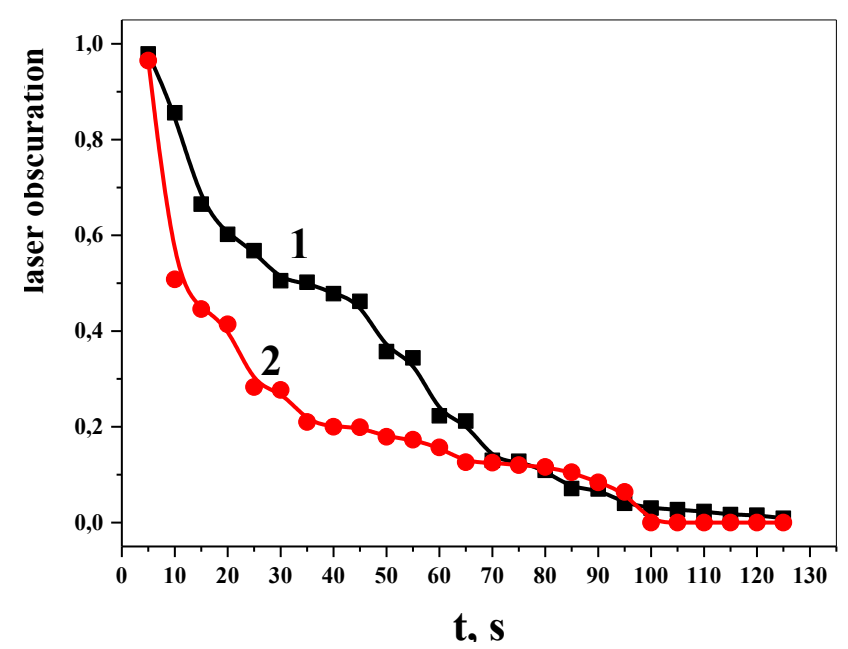

Fig. 5: Kinetics of NAM dissolution before (1) and after (2) fine grinding process ( $n=3, P=0,95)$

The reaction rate constant was determined by the coefficient $b$ of the linearized section of the straight line as the tangent of the angle of inclination to the abscissa axis:

$$
\begin{aligned}
& k=-\operatorname{tg} \alpha \\
& \operatorname{tg} \alpha=-d \text { laser obscuration/dt }
\end{aligned}
$$

The degree of pharmaceutical substances fine grinding determines the rate of dissolution, absorption and drug therapeutically effect. Our results show a twofold increase in the NAM dissolution rate constant after fine grinding compared to the native sample: $\mathrm{k}=0,026 \mathrm{~s}^{-1}$ and $\mathrm{k}=0,040 \mathrm{~s}^{-1}$ for native and MA samples of nocotinamide. This may directly indicate that the therapeutic efficiency, absorption rate permeability and withdrawal of the drug.

\section{Conclusion}

Thus, studies have shown that mechanical activation by grinding in a knife mill of the type of "free, direct impact" is accompanied by a change in the properties and increased biological activity of nicotinamide, as known in the therapy of prevention of photoageing and skin neoplasia. This has been demonstrated by changing the activation energy of the 
cell's transition to a "dead cell" state and increasing the rate of the chemical process of dissolving mechanoactivated molecular nicotinamide crystals in water. The results of the study can be used in medicine to improve the efficiency and reduce doses of drug therapy.

\section{Acknowledgements}

The publication has been prepared with the support of the "RUDN University Program 5-100".

\section{Referenses}

1.Y. Zhang, J. Yu, H. Bomba, Y.Zhu, Z.Gu, Chem. Rev., 116, 12536-12563 (2016)

2.K.H. Vining, D.J. Mooney, Nat Rev Mol Cell Biol., 18, 728-742 (2017)

3.J. Wang, J. A. Kaplan, Y. L. Colson, M. W. Grinstaff, Adv Drug Deliv Rev, 108, 68-82 (2017)

4.European journal of pharmaceutics and biopharmaceutics: official journal of Arbeitsgemeinschaft fur Pharmazeutische Verfahrenstechnik e.V, 56, 393-400 (1991)

5.A. Syroeshkin, E. Uspenskaya, T. Pleteneva, M. Morozova, T. Maksimova, A. Koldina,

M. Makarova, O. Levitskaya, I. Zlatskiy, Int J App Pharm, 11, 118-123 (2019)

6.A.K. Aman, R.K. Singh, R. Kumar, A.K. Ghos, IJPSR, 9, 672-677 (2018)

7.A. Majumdar, N. Dubey, Int J App Pharm, 11, 188-193 (2019)

8.P.S. Reddy, K. A. Suresh, V. Jain,. Int J App Pharm, 10, 54-59 (2018)

9.L. Fania, C. Mazzanti, E. Campione, E. Candi, D. Abeni, E. Dellambra, Int J Mol Sci., 20, 5946 (2019)

10.R.A. Fricker, E.L. Green, S.I. Jenkins, S.M. Griffin, Int J Tryptophan Res, 11, $1178646918776658(2018)$

11.M. Knip, I.F. Douek, W.P.T. Moore, H.A. Gilmor, A.E.M. McLean, P.J. Bingley, E.A.M. Gale, Diabetologia, 43, 1337-1345 (2000)

12.A. Hoffer, Biochemistry of nicotinic acid and nicotinamide. Psychosomatics, 8, 95-100 (1967)

13.V. Plotnikov, V. Kuznetsova, The Prospects for the Use of Digital Technology "Blockchain" in the Pharmaceutical Market, MATEC Web of Conferences, 193, 02029 (2018)

14.W. König, R. Komanduri, H.K. Tönshoff, G. Ackershott, CIRP Annals, 33, 417-427 (1984)

15.G. Rumpf, On the main physical issues in grinding. Frankfurt am Main: European meeting on grinding, 8, 95-100 (1962)

16.B. Gurrutxaga-Lerma, D.S. Balint, D. Dini, D.E. Eakins, A.P. Sutton, Proc R Soc A, 469, 20130141 (2013)

17.A.M. Koldina, E.V. Uspenskaya, A.A. Borodin, T.V. Pleteneva, A.V. Syroeshkin, Int J App Pharm, 11, 271-278 (2019)

18.European Pharmacopoeia 8.0 (1-2), (Strasbourg: Council of Europe, 2014)

19.L.F. Bilenko, Int J Miner Process, 5, 3-6 (1997)

20.V.V. Goncharuk, A.V. Syroeshkin, I.A. Zlatskiy, E.V. Uspenskaya, Orekhova, O.V. Levitskaya, V.I. Dobrovolskiy, T.V. Pleteneva, J. of Water Chemistry and Technology, 39, 97-102 (2017) 